\title{
Quelles stratégies pour dire l'espace dans un guide de voyage ? L'entrée « Lausanne » dans quelques guides entre 1714 et aujourd'hui
}

\section{Ariane Devanthéry}

\section{CpenEdition}

Journals

Édition électronique

URL : http://journals.openedition.org/edl/486

DOI : $10.4000 /$ edl.486

ISSN : 2296-5084

Éditeur

Université de Lausanne

\section{Édition imprimée}

Date de publication : 15 mai 2013

Pagination : 91-108

ISBN : 978-2-940331-32-1

ISSN : 0014-2026

\section{Référence électronique}

Ariane Devanthéry, «Quelles stratégies pour dire l'espace dans un guide de voyage ? L'entrée

«Lausanne » dans quelques guides entre 1714 et aujourd'hui », Études de lettres [En ligne], 1-2 | 2013, mis en ligne le 15 mai 2016, consulté le 18 décembre 2020. URL : http://journals.openedition.org/edl/ 486 ; DOI : https://doi.org/10.4000/edl.486 


\section{QUELLES STRATÉGIES POUR DIRE L'ESPACE DANS UN GUIDE DE VOYAGE? \\ L'ENTRÉE "LAUSANNE» DANS QUELQUES GUIDES ENTRE 1714 ET AUJOURD'HUI}

Si les récits de voyage racontent un périple particulier, achevé et non exactement duplicable, les guides de voyage doivent dire une multitude de voyages futurs. Les premiers sont ainsi récits du passé, tandis que les seconds sont descriptions de possibles. Quelles ont été les stratégies des auteurs de guides pour décrire une ville ou un trajet en laissant de la place aux futurs voyageurs? Un guide très subjectif présente-t-il l'espace de manière très différente qu'un guide voulu neutre? Sachant que l'importance de la présence d'un auteur de guide dans son texte a varié entre le XVIII ${ }^{\mathrm{e}}$ siècle et aujourd'hui, quelle(s) différence(s) constate-t-on dans les manières de dire l'espace? On cherchera des réponses en lisant l'article consacré à Lausanne dans divers guides d'hier et d'aujourd'hui.

\section{Dire des possibles}

Les études littéraires ont mis du temps à s'intéresser aux guides de voyage. Certains doutaient, en effet, que ce type de texte ait un quelconque lien avec la littérature. Même si l'on peut rarement attribuer de réelles prétentions esthétiques à ces textes (prétentions que leurs auteurs ne revendiquent d'ailleurs pas), on ne peut nier qu'ils font partie d'une large littérature de voyage et qu'il est possible de leur appliquer une lecture de type littéraire. Mais, avant cela, une mise au point théorique est utile.

Les guides de voyage sont des textes particuliers que l'on ne peut associer aux récits de voyage. Si les récits de voyage racontent en effet des 
périples singuliers, achevés et non exactement duplicables, les guides de voyage doivent dire une multitude de voyages futurs. Les premiers sont ainsi récits du passé, d'un voyage unique qui ne sera jamais exactement reproductible, tandis que les seconds sont descriptions de possibles, propositions d'espace et de temps à remplir par l'expérience des voyageurs. Sur une même base, une expérience vécue dans le monde physique, les uns disent ce qui a été tandis que les autres cherchent à jalonner ce qui sera ou pourrait être.

\section{Une grille de lecture pour les guides}

A cette différence première, il faut ajouter que les guides de voyage ont, contrairement aux récits de voyage qui peuvent travailler une matière utopique ou imaginaire, une fonction fondamentalement pragmatique. Un guide doit en effet être utile à son lecteur, répondre à ses demandes de connaissance autant que d'informations pratiques, en lui permettant de mieux connaître l'histoire ou la géologie d'une région comme de trouver un hôtel. Les auteurs-concepteurs de guides de voyage ont compris depuis longtemps qu'ils s'adressaient à un double public d'utilisateurs ayant des besoins très divers. Les voyageurs d'une part, qui doivent pouvoir se déplacer dans un espace qu'ils ne connaissent pas, et les lecteurs d'autre part, qui ont eux aussi des besoins particuliers. Celui d'abord de pouvoir circuler aisément dans le guide-livre pour trouver ici une information encyclopédique sur l'histoire générale du pays, là une recommandation d'hôtel et ailleurs la description précise d'une route; mais aussi celui de pouvoir pratiquer une lecture discontinue, qui est assez typique de l'usage d'un guide en situation. Faite sur les lieux de visite, cette lecture implique que l'œil quitte très souvent le texte du guide pour vérifier si/comment les informations lues sont réalisées dans le monde. La statue de la Justice qui surmonte cette fontaine compte-t-elle bien quatre petits personnages à ses pieds? Le moment du retour au texte est tout à fait déterminant: l'œil doit pouvoir retrouver rapidement la ligne qu'il a quittée et reprendre le fil de sa lecture. Pour répondre à la particularité de cette lecture non linéaire, les concepteurs de guides ont choisi de "marquer" les textes typographiquement, en faisant ressortir en caractères gras ou italiques, en petites capitales ou en couleur, certains mots. Agissant comme des repères dans l'écrit, ils sont autant 
d'ancrages pour une lecture vagabonde ${ }^{1}$. Tout cela concourt à la mise en place d'une communication originale.

Ce triple repérage (dans le monde physique, dans les pages d'un livre et au sein du texte) constitue une fonction propre aux guides de voyage, la fonction "pratique». Elle a connu de grands changements au début

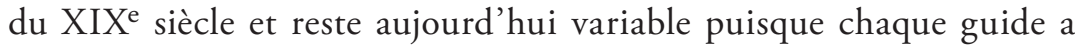
ses propres règles ${ }^{2}$. Elle constitue une part importante de la grille de lecture que j'ai dû élaborer pour étudier les guides de voyage en m'attachant à leur propre apport et non à ce que j'aurais voulu y trouver, ou en me basant sur les codes des récits de voyage. Trois autres fonctions la complètent: la fonction épistémique (qui prend en compte les connaissances transmises et qui est essentielle à tout guide de voyage), la fonction esthétique culturelle (qui s'attache à la diffusion des modèles culturels, tels le classicisme, le romantisme ou la post-modernité) et enfin la fonction testimoniale. Cette dernière fonction est importante quand on cherche à identifier les stratégies pour dire l'espace, car elle est la trace du voyageur-témoin, de celui qui a fait le voyage en premier et qui en propose une mise en forme. On verra qu'elle a connu et continue à connaître d'importants changements. Elle est directement liée à la posture auctoriale. Selon les périodes ou, pour l'époque contemporaine, suivant le choix éditorial, la présence de l'auteur dans son texte a beaucoup varié. Forte et habituelle au XVIII ${ }^{\mathrm{e}}$ siècle $^{3}$, elle tend à s'effacer au profit d'une claire recherche d'objectivité et de neutralité dans la seconde moitié du XIX ${ }^{\mathrm{e}}$ siècle et une grande part du $\mathrm{XX}^{\mathrm{e}}$ siècle; depuis les années 1970, elle est libérée, laissant à l'auteur-concepteur de guide contemporain le choix de son ton.

Définissons enfin les guides de voyage: je propose de les considérer comme des accès. Ils offrent en effet des accès à un espace inconnu, à un savoir sur cet espace et à des possibilités d'émotions. Sans nous étendre trop longuement sur ce dernier point, précisons qu'un guide doit dire

I. Les dictionnaires et les encyclopédies présentent le même cas de figure.

2. C'est elle qui fait qu'un guide doit en général donner son propre "mode d'emploi ".

3. Citons ici l'Avertissement de François-Maximilien Misson, auteur d'un des plus grands guides de l'Italie de la première moitié du XVIII ${ }^{\mathrm{e}}$ siècle pour les voyageurs parlant français: «Je déclare que je n'oublie rien, puisque je ne promets rien précisément. [...] J'en dis ce que j'en ay vu, ce que j'en ay appris de gens dignes de foy, \& ce que je trouve à propos d'en dire.» F.-M. Misson, Nouveau voyage d'Italie, sans pagination. 
que, si le voyageur se rend à tel endroit, il pourra ressentir un vertige physique (il est en montagne), historique (il est sur un champ de bataille célèbre) ou esthétique (Turner, Goethe ou Hugo sont passés ici). Mais il ne devra pas aller au-delà de la simple information. Car, à ce moment, non seulement le guide empièterait sur le travail du voyageur (c'est en effet à lui de ressentir et d'actualiser l'émotion), mais il serait aussi en train de tendre vers le récit de voyage. Stendhal avait compris cette distinction, lui qui a noté dans son journal en 1813:

Un journal de voyage doit être plein de sensations, un itinéraire [= un guide de voyage] en être vide. Il doit dire: à Saint-Pierre in Montorio, voir l'Assomption du Guide, peinte en 1553, payée 38 écus au peintre, qui avait alors trente-sept ans.

Le mélange de la sensation avec l'indication est détestable et diminue infiniment le plaisir du voyageur qui se trouve en présence de ce qu'un autre homme a senti, au lieu d'être livré à son propre sentiment ${ }^{4}$.

\section{Dire l'espace}

Corollaire de leur rôle fondamentalement pragmatique, les guides de voyage ont un très fort lien avec le monde physique. Loin du rêve, ils sont contraints de n'évoquer que l'existant et le réalisable. La rapidité de leur péremption, de ce temps où ils ne sont plus des reflets assez fidèles du monde, est le signe de l'étroitesse du lien. Ils ont en revanche un pouvoir que les récits de voyage ne possèdent guère: en proposant des actions à réaliser dans l'espace physique (s'arrêter à un point de vue, visiter telle grotte ou abbaye), ils ont potentiellement la possibilité d'induire des répercussions concrètes, comme, par exemple, la création d'un nouvel accès ou l'agrandissement d'un parking.

Echos des voyages eux-mêmes, deux grands types d'espace se retrouvent dans les guides: celui du trajet, d'une description en mouvement, et celui de la pause ou des lieux, qui implique souvent une description à l'arrêt ${ }^{5}$. Echos des strates des déplacements, ces descriptions peuvent se faire à l'échelle du voyage comme à l'échelle d'un lieu, au sein d'une ville ou d'un parcours à l'intérieur d'un château. Face à cette diversité,

4. Stendhal, Journal, t. 1, p. 878. L'exemple semble être fantaisiste.

5. Voir M. de Certeau, «Récits d'espace» et M. Desportes, Paysages en mouvement. 
les auteurs de guides ont utilisé deux modèles pour mettre en forme ces espaces: l'itinéraire (le meilleur moyen pour retracer un déplacement, qu'il soit boucle ou "route» ${ }^{6}$ ) et l'ordre logique, qu'il soit alphabétique, par monuments ou suive n'importe quel autre schéma.

L'expérience de l'espace étant primordiale pour tout voyageur ${ }^{7}$, sa mise en forme est donc essentielle pour tout guide de voyage. Sur la base des remarques faites plus haut, de nombreuses questions se posent. Quelles ont été et sont les stratégies des auteurs de guides pour décrire une ville ou un trajet tout en laissant de la place aux futurs voyageurs? Un guide très subjectif présente-t-il l'espace de manière très différente qu'un guide voulu neutre? Sachant que l'importance de la présence d'un auteur de guide dans son texte a varié entre le début du XVIII e siècle et aujourd'hui, quelle(s) différence(s) constate-t-on dans les manières de dire l'espace? J'ai cherché des réponses à ces questions en lisant l'article consacré à Lausanne dans une vingtaine de guides d'hier et d'aujourd'hui. Le format de cet article fera cependant que seuls trois guides seront présentés.

\section{Lecture diachronique}

4. 1. Le Guide des voyageurs en Europe, H. A. O. Reichard, 1793

Conseiller de guerre du duc de Saxe-Gotha, Heinrich August Ottokar Reichard (1751-1828) a été un grand voyageur autant par métier que par goût. Il a publié en 1784 un premier Handbuch für Reisende aus allen Ständen, qui a été traduit en français en 1793 sous le nom de Guide des voyageurs en Europe. Voici l'extrait présentant le trajet de Payerne à Genève, via Lausanne:

6. La mise en forme par "route» est l'un des grands apports des guides du XIX ${ }^{\mathrm{e}}$ siècle: une "route» est une fraction d'un itinéraire plus long. Les trajets GenèveLausanne, Lausanne-Martigny, Martigny-Simplon constituent trois routes que l'on peut ensuite associer librement.

7. Voir A. Dévanthery, Itinéraires; «Entre espaces perçus et espaces à percevoir»; J. Dewitte, «Espace du paysage et espace géographique»; C. Reichler, "Le marcheur romantique et la phénoménologie du chemin». 
A Payerne on regagne la grande route de Geneve. Moudon est le Minodunum des Romains, comme l'apprend une inscription romaine qu'on a fait enchasser sur la porte de la maison de ville. Du sommet de la montagne, que l'on commence à grimper, en sortant de Moudon, on apperçoit pour la première fois les Alpes de la Savoie, et même le Mont-blanc.

Lausanne. V. tableau. L'église de Morges est joliment située. A Rolle on peut se détourner pour voir Aubonne, célèbre par ses belles vues surtout dans un lieu nommé le Signal de Bougy. [...] La ville de Nyon est très-ancienne. [...] Près du château il y a une promenade charmante; il $\mathrm{y}$ a aussi une manufacture de belle porcelaine [...].

A Genève commencera la troisième et dernière excursion dans les Alpes que je proposerai aux voyageurs ${ }^{8}$.

On l'a dit: les guides du XVIII e siècle sont généralement plus subjectifs que ceux de 1900. Commençons donc par lister les éléments qui dénotent cette subjectivité. Bien présente, celle-ci se retrouve lors d'occurrences variées: dans la formule "je proposerai»; dans les multiples termes axiologiques qui attestent d'une actualisation des émotions par l'auteur du texte ("joliment», "charmant», "belle»); dans la rapide suite d'informations, dont la sélection tient probablement autant aux vogues esthétiques du temps qu'aux choix personnels de l'auteur; dans la présentation enfin de l'espace sous la forme d'un trajet unique (on va de Payerne à Genève et les autres routes ne sont pas considérées), ce qui rend de facto le guidage lacunaire. Mais, auteur d'un guide de la fin du XVIII ${ }^{\mathrm{e}}$ siècle, soit dans une période de transition vers la forme "moderne» des guides, Reichard cherche aussi à prendre en compte les voyageurs dans leur diversité. Il compose donc son texte en "on" et au présent (mode accueillant pour les lecteurs-voyageurs) et il leur laisse une place en intégrant des possibilités de variantes ("on peut se détourner»). La posture auctoriale de Reichard est donc mixte: souvent encore voyageur-témoin, mais aussi, parallèlement, auteur de guide cherchant à laisser leur place aux futurs voyageurs. Les noms de lieux étant imprimés en italiques, on réalise que des éléments matériels, tels la typographie, participent aussi à la prise en charge du déplacement.

Un point aura peut-être surpris: n'est-ce pas étonnant qu'au moment de décrire Lausanne, la grande ville de ce trajet, on tombe sur du vide?

8. H. A. O. Reichard, Guide des voyageurs en Europe, 1793, t. 1, p. 540. 
"Lausanne. V. tableau.» Cet effet de blanc vient du choix qu'a fait Reichard d'associer dans son guide deux manières différentes de présenter l'espace. En utilisant en effet deux modèles concurrents, l'itinéraire et l'alphabet (les villes importantes sont décrites à part et par ordre alphabétique, au début du guide), il crée pour son lecteur l'obligation de circuler dans le guide et d'aller chercher une centaine de pages plus tôt les informations dédiées à Lausanne. Le fait que la description du trajet entre ici en conflit avec la description des lieux indique que les deux modèles de présentation de l'espace que sont l'itinéraire et l'ordre logique ne sont pas toujours faciles à manier.

Les guides de voyage n'ont toutefois pas que leur texte imprimé pour parler d'espace. Une fois que cela est devenu possible techniquement, leurs auteurs-concepteurs leur ajoutent volontiers des compléments graphiques, cartes, plans ou images (gravures ou photographies). Le fait que Reichard propose plusieurs cartes dans son guide dès 1793 souligne fortement sa claire prise en compte du voyageur et de sa liberté. Avec une bonne carte, on est en effet moins dépendant du texte d'un guide et l'on peut envisager de dessiner son propre cheminement. A partir du début du XIX ${ }^{\mathrm{e}}$ siècle, les cartes topographiques et routières, puis touristiques se généralisent progressivement et commencent à fonctionner régulièrement en tandem avec le texte des guides pour dire l'espace?

Après nous être intéressés à la manière dont Reichard décrit l'arrivée à Lausanne, voyons ce qu'il dit de la ville:

LAUSANNE. Population, 7 à 8'000 h.

Edifices remarquables. Curiosités. L'église cathédrale (elle renferme les tombeaux de la princesse Orlow, et de la duchesse de Courlande) - le collège - l'hôtel de ville - l'arsenal - l'hôpital - quelques restes d'antiquités [...] - (Le célèbre docteur Tissot, qui n’a pas moins contribué d'illustrer cette ville, loge à Montrion, à sa maison de campagne.) Promenades. La terrasse près de l'église cathédrale (la vue est des plus étendues); la promenade de Monbenon; à une demi-lieue au-dessus de la ville est un rocher élevé, nommé le Signal, qui offre de superbes perspectives; l'un des plus brillans endroit c'est Belle-vue; Beau-lieu,

9. Faute de place, je n'entrerai pas plus avant dans cette discussion ici. Mais on peut consulter A. Devanthéry, «Entre itinéraires et trajets, représentations des déplacements dans les guides de voyage au tournant du XIX ${ }^{\mathrm{e}}$ siècle» ou A. Devanthéry, Itinéraires. 
campagne que M. Necker a habitée, n'est pas moins remarquable par sa situation ${ }^{10}$.

Cet extrait transcrit la première des quatre pages que le guide consacre à Lausanne. L'article est construit par une suite d'entrées thématiques: "édifices remarquables, curiosités", "promenades", "fabriques", "établissements littéraires et utiles", "collections, cabinets", "auberges", etc. A l'intérieur de ces parties, l'espace est évoqué sous forme de liste, d'énumération. On le constate, les lieux à voir sont répertoriés successivement et de manière autonome, sans être reliés les uns aux autres, et simplement séparés typographiquement par un long tiret. Si l'on cherche à réaliser l'itinéraire que ce catalogue dessine, on se rendra compte que le trajet à réaliser en ville de Lausanne est complètement erratique. L'énumération des promenades possibles, qui fait passer de la terrasse de la cathédrale à Montbenon, au Signal, à Bellevue pour finir à Beaulieu est un très bon exemple de cette errance pour quiconque connaît un peu la topographie lausannoise. On n'est donc clairement pas dans un modèle de représentation de l'espace qui prend en charge la description des trajets, qui indique comment aller de l'hôtel de ville à l'arsenal, mais bien dans celui d'une énumération logique. Comme la description des localisations et des trajets manque, les formules de localisation (au nord, à gauche, derrière l'église) sont aussi absentes.

\subsection{La Suisse, Karl Baedeker, 1905}

Vers 1900, les guides Baedeker étaient tellement connus que le nom "Baedeker» était devenu un nom commun, synonyme de "guide». Ce sont les guides emblématiques du tourisme Belle Epoque. On y lit une évidente volonté de neutralité et une claire recherche d'objectivité, ce qui signe la quasi-disparition de la fonction testimoniale. A l'opposé d'une présentation personnelle des lieux, les auteurs ont souhaité laisser beaucoup de place au voyageur et à ses choix. De nombreuses formulations attestent en effet du désir de dire sans contraindre ("on peut», "il y a», «il est possible», ainsi que les tournures modélisées ou impersonnelles). Alors que le Baedeker de 1901 (22 édition) présentait encore Lausanne en partant de la cathédrale et proposait plus une suite de monuments à voir qu'un réel trajet en ville, suivant en cela une idée logique et une

Io. H. A. O. Reichard, Guide des voyageurs en Europe, 1793, t. 1, p. 442. 
hiérarchie des lieux à visiter (videnda), celui de 1905 (24e édition) propose un tour complètement repensé et centré sur le visiteur-marcheur. Celui-ci part en effet de la place St-François (le plus logique quand on se situe dans la perspective d'un piéton arrivant à Lausanne) et décrit attentivement les cheminements. Citons rapidement un extrait de sa présentation de la ville:

Lausanne (483 m.) [...]

La Place de St-François (pl. C4), avec l'église du même nom (bâtie en 1442 et restaurée de 1898 à 1903), le bel hôtel des postes et la banque cantonale, est le centre des tramways électriques. Au N.-E., on descend par la rue fortement inclinée de St-François et remonte ensuite la rue du Pont à la place de la Palud (pl. C3), la place du marché, où l'on voit l'hôtel de ville (1454) et la vieille fontaine de la Justice (1557). De là, on monte à dr. par des rues tortueuses ou bien à g. par les Escaliers-duMarché (escalier couvert de 160 marches) à la terrasse de la cathédrale (529 m.), l'anc. cimetière, d'où l'on découvre un beau panorama de la ville, du lac et des Alpes de Savoie ${ }^{11}$.

Aussi construit en «on" et au présent, le texte propose un guidage très développé. On lit en effet de nombreuses formules de repérage dans l'espace que les verbes de mouvement ("on descend [...] et remonte») et une idée de successivité ("ensuite») viennent appuyer. Les formules de localisation ("au N.-E.», "de là" et "à dr. [...] ou bien à g.") sont nombreuses et définissent trois types différents de rapport à l'espace. Le premier, absolu, utilise les points cardinaux; le deuxième, relatif mais rapporté à un positionnement fixe (telle une église ou une fontaine), use de formules du type de: "à proximité de, au-delà de, au-dessus de..."; et le dernier est un positionnement subjectif, qui est rapporté au corps en mouvement: "à droite....à gauche». Il implique soit une forte présence du voyageur-témoin initial, soit un important travail opéré par les auteurs-concepteurs du guide pour faciliter les déplacements aux futurs voyageurs en se mettant à leur place. $\mathrm{Si}$, parfois, le guidage manque, c'est que l'édifice présenté est tout proche du précédent, ou parce que l'on fait appel au plan de ville pour aider à le localiser (l'extrait ci-dessus fait par deux fois référence au plan de Lausanne intégré à l'article: "place de la Palud (pl. C3)»). On peut en effet relever comme une particularité des

II. K. Baedeker, La Suisse et les parties limitrophes de la Savoie et de l'Italie, p. 289. 
guides Baedeker de cette époque une tendance à la "sur-localisation": "Sur la place de la Riponne (pl. C3), à l'O. au-dessous de la terrasse de la cathédrale...» ${ }^{12}$. En un temps où les auteurs de Baedeker cherchent surtout à condenser l'information pour réduire le volume de leur guide et malgré la place évidente que prend, comme ici, la quadruple indication d'un lieu, quelqu'un a considéré ce que cela en valait la peine. Cette "sur-localisation" peut ainsi être considérée comme un élément de mesure permettant d'évaluer l'importance que le guidage dans l'espace avait alors pris dans ces guides.

\section{3. Guide du Routard, La Suisse, 2006}

Si les guides du XVIII ${ }^{\mathrm{e}}$ siècle étaient majoritairement subjectifs et que les guides de la fin du XIX et de la première moitié du $\mathrm{XX}^{\mathrm{e}}$ siècle recherchaient la neutralité et voulaient clairement effacer la présence du voyageur-témoin ${ }^{13}$, les auteurs-concepteurs des guides de voyage d'aujourd'hui ont le choix. Le curseur peut donc se trouver à tout endroit de cette échelle. La conséquence est que les rayons des librairies débordent de guides typés ou plus lisses, proposant une grande variété de formes et de ton. Ce dernier peut être le fait d'un auteur particulier, mais il est souvent celui d'une maison d'édition, qui a défini une ligne éditoriale dont le ton fait partie. On peut d'ailleurs remarquer qu'une même maison d'édition a souvent des collections au ton très différent (pensons aux guides Gallimard «Encyclopédies du voyage» et "Cartovilles» ou aux guides Hachette "Voir» et «Le Routard»).

Pour tester un guide typé d'aujourd'hui, arrêtons-nous sur le Guide du Routard. Publié pour la première fois en 1997 (Philippe Gloaguen pensait jusqu'alors qu'il était impossible de proposer un guide de la Suisse destiné aux petits budgets), il a été rédigé par deux Helvètes: Pierre-Alain Rattaz et Christian Chevrolet:

I2. Ibid., p. 290.

13. C'est le cas des trois grands guides culturels généralistes de cette époque: le Baedeker allemand, le Joanne français et, quoique dans une moindre mesure, le Murray anglais. 
C'est la première fois qu'un de nos guides est entièrement réalisé par des locaux. On a bien sûr tout relu. Il faut que ça rentre dans la ligne du Routard ${ }^{14}$.

Une ligne éditoriale qui donne bien sûr le ton. Quand il présente Lausanne, le Routard met en œuvre deux logiques différentes: celle qui glisse de proche en proche au centre ville et une logique de liste et de sélection pour la périphérie. Citons-en un extrait:

*La place Saint-François (plan C1, 80): les promeneurs vont vite le comprendre, Lausanne n'est qu'une succession de montées et de descentes... Alors au centre, tout en haut, la place Saint-François. Celle qui fut vraisemblablement l'une des plus belles places du pays a été joyeusement saccagée au cours du XXe siècle. [...].

* La place de la Palud (plan C1, 79): en descendant la rue commerçante et piétonne Saint-François, puis en remontant la rue du Pont [...], on rejoint la place de la Palud. [...].

*** La cathédrale (plan C1, 78): au début de la rue de la Mercerie, prendre à gauche et monter par les très beaux escaliers de bois couverts pour rejoindre la cathédrale. [...] Le plus grand et le plus bel édifice gothique de Suisse. Attention, chef-d'œuvre, même s'il lui manque une tour! [...].

Allez, on entre! Et l'on en reste complètement abasourdi... ${ }^{15}$

Les localisations sont clairement bien meilleures que celles du guide de Reichard, mais elles sont aussi moins précises que celle du Baedeker du début $\mathrm{du} \mathrm{XX}^{\mathrm{e}}$ siècle. Très centrées sur le corps du voyageur, elles sont parfois étranges, notamment quand elles ne donnent pas le lieu qui leur sert de référence ("Alors au centre, tout en haut, la place SaintFrançois»). Si «au centre» peut assez facilement être complété par «de la ville» ou "du plan présent dans le guide», le «tout en haut» est beaucoup plus étonnant, la place Saint-François n'étant de loin pas le lieu le plus élevé de Lausanne. Même type d'erreur de localisation (et donc, par ricochet, de guidage) à la cathédrale: le texte laisse entendre que

I4. Voir Archives de la Tribune de Genève (10 mars 1997) : «Le célèbre Routard franchit enfin la frontière suisse».

I5. Le Guide du Routard, Suisse, p. 123 sq. J'ai remplacé par des astérisques le symbole rouge du petit routard qui code l'importance des lieux; ils fonctionnent en effet de la même manière. 
l'on rentre dans l'église par le portail peint au sud de l'édifice, alors que l'entrée se trouve à l'ouest. Au chapitre des contrevérités, ce guide n'est pas à l'abri non plus d'erreurs concernant les connaissances transmises: Lousonna a été fondée en - 15 et non « 3 siècles av. J.-C.» et la Salle du Grand Conseil a brûlé en 2002.

De manière générale, le guidage dans Lausanne du Routard est ainsi plutôt médiocre. Un "effet de liste» est souvent présent, heureusement atténué par l'évocation des lieux de proche en proche ou par une référence au plan pour les endroits plus éloignés (relevons toutefois que certains buts de visite proposés - la tour Haldimand ou Vidy, par exemple - sont hors du plan du guide). Je précise que je ne cherche pas ici à juger le Routard, mais simplement à comprendre les procédés de guidage mis en place dans son texte et sa structure (les plans et cartes, l'organisation des chapitres ou des index et même sa typographie sont à considérer). Si les "vides» identifiés dans le guidage peuvent ainsi être perçus comme autant de lacunes, reconnaissons qu'ils peuvent aussi être vus comme des éléments positifs, précisément parce qu'ils laissent de la place (et donc de la liberté) aux voyageurs. Une liberté que l'éditeur du Routard met d'ailleurs régulièrement en avant et que ses lecteurs-utilisateurs revendiquent fréquemment. Le site internet du Routard l'affirme: «le GDR s'est toujours attaché à refléter un mode de vie particulier, synonyme de liberté, d'insolence et de curiosité» ${ }^{16}$.

Si l'on s'attache cependant à son ton, il en ressort un constat contradictoire. Parce qu'il est rédigé d'une manière résolument subjective (qui signe ici le grand retour de la présence du voyageur-témoin), la liberté du lecteur est paradoxalement moindre. Pour ce qui concerne la langue, c'est en effet le Routard qui use des formules les plus contraignantes, mélange d'impératifs et de points d'exclamation («Allez, on entre!») ou de sens impératif ("Une petite visite s'impose»). Les jugements personnels sont aussi nombreux ("toutes les heures, animations complètement kitsch de l'horloge juste à côté») et parfois non fondés historiquement (comme à propos de la place Saint-François: «celle qui fut vraisemblablement l'une des plus belles places du pays a été joyeusement saccagée au cours du $\mathrm{XX}^{\mathrm{e}}$ s.»). De même, l'émotion est souvent actualisée, c'est-à-dire donnée via la perception personnelle d'un auteur ("Attention, chef-d'œuvre

I6. http://www.routard.com/planete_coulisse/page/saga.htm, consulté le 28.04.2011. 
même s'il lui manque une tour! [...] Et l'on reste complètement abasourdi...»). On le constate, ce que les concepteurs des guides Baedeker ont précisément travaillé à effacer, la présence d'un voyageurauteur-témoin, est ici non seulement affichée, mais revendiquée. A moins que l'on ne compte sur un hypothétique "effet miroir" qui tablerait sur l'idée qu'un voyageur qui lit un texte subjectif se sentirait en retour dédouané et, par là même, autorisé à se montrer lui aussi subjectif, ce guide, qui se veut résolument libre et s'adressant à des voyageurs ${ }^{17}$ eux aussi résolument libres, le fait de manière bien contraignante.

\section{Les guides, entre guidage et témoignage}

Au terme de cette triple lecture, peut-on apporter une réponse claire à la question qui a initié cette enquête? La manière de présenter l'espace dans un guide varie-t-elle suivant le degré de subjectivité du texte? Comme les Normands, on répondra et oui et non.

Non d'abord, pour ce qui touche aux trois types de rapports à l'espace que l'on a relevés, une forte subjectivité ne semblant pas avoir ici de conséquence. Qu'ils soient absolus (usage des points cardinaux), relatifs (localisés par rapport à un monument ou un lieu précis) ou subjectifs (la situation se fait par rapport au corps du voyageur-lecteur), ces différents rapports à l'espace sont présents dans toutes sortes de textes et ne peuvent être utilisés pour distinguer un récit de voyage (auquel on attribuerait une valeur principalement testimoniale) d'un compte-rendu d'expédition scientifique qui, lui, pourrait présenter idéalement un aspect plus objectif et épistémique. L'usage de formules de localisation géographique très subjectives (à gauche, à droite) ou plus objectives (au nord) dépend en effet moins du type de texte où on les trouve que du rapport à l'espace que l'auteur veut établir: essentiellement logique ou très proche du corps.

Mais oui pour ce qui est du guidage. Il semble bien, en effet, qu'un lien puisse être établi entre un guidage dans l'espace réussi et le degré de subjectivité du texte du guide. Quand la présence de l'auteur-voyageur est clairement sensible, le guidage est en effet souvent médiocre et présente volontiers un "effet de liste», alors que quand l'auteur a adopté

17. Et non des touristes, ceux-ci étant par définition considérés dans le Routard comme des voyageurs au rabais. 
une posture neutralisée, le guidage semble meilleur. Ce résultat n'est cependant encore qu'une tendance, qui paraît de plus paradoxale. Il serait ainsi bon de confirmer l'étude et que d'autres lecteurs interrogent d'autres guides qui évoquent d'autres endroits. Le constat selon lequel un texte de guide fortement imprégné de subjectivité semble impliquer une moins bonne prise en compte des voyageurs ne dit toutefois rien des lieux, qui, eux, sont souvent très bien traités.

La lecture diachronique à laquelle on s'est rapidement livré a aussi fait ressortir que la relation entre les guides et les lieux décrits avait connu de grands changements en quelque trois cents ans et qu'elle devait être historicisée. Alors qu'au XVIII siècle, cette relation était perçue comme transparente (malgré sa modélisation par la perception de l'auteurvoyageur), elle est devenue plus complexe au XIX ${ }^{\mathrm{e}}$. Si les phénomènes d'intertextualité et de critique étaient déjà très présents au XVIII ${ }^{e}$ siècle, la grande vogue du tourisme romantique, qui a banalisé les lieux autant que les émotions par une trop grande diffusion, a aussi introduit progressivement un nouvel élément jusque-là peu présent dans les guides de voyage: l'ironie. Une ironie, qui, comme un voile, vient s'interposer entre le monde physique et les textes qui l'évoquent. Aujourd'hui, la relation qu'entretiennent les guides de voyage aux lieux décrits est clairement complexe. La plupart des guides, qui affirment leur liberté à décrire le monde de façon critique, le proposent donc filtré. Si le futur lecteur-voyageur trouve utile ou nécessaire de connaittre le type de regard qu'il achète avec son guide, il lui faut se référer au positionnement de la Maison d'édition, positionnement qu'elle transmet souvent au travers de sa publicité. Mais pas toujours. On peut donc déplorer un certain manque de transparence sur cette question et souhaiter plus de clarté.

Il reste qu'aucune description du monde n'est neutre. Et que celles que proposent les guides, comme toutes les autres, doivent être problématisées, interprétées et lues de manière critique. Contrairement à ce qu'on aimerait peut-être croire, les guides de voyage ne permettent en effet pas un accès transparent au monde, car ce sont aussi des constructions qui obéissent à des codes. Du voyageur-témoin du XVIII siècle au voyageur-observateur ou arbitre du monde du tourisme (ce qu'ont construit les éditions Baedeker dans la seconde moitié du XIX siècle), c'est la posture auctoriale qui est ici en jeu ${ }^{18}$. La fonction de l'auteur de guide

I8. J. Meizoz, «Ethos et posture d'auteur (Rousseau, Céline, Ajar, Houellebecq)». 
est ainsi peut-être la seule qui ne change pas réellement: il est et reste un passeur de connaissances.

Ariane DevanthéRY

Université de Lausanne 


\section{BIBLIOGRAPHIE}

\section{Sources}

Archives de la Tribune de Genève, http://archives.tdg.ch/TG/TG/-/ article-1997-03-29/le-celebre-routard-franchit-enfin-la-frontieresuissele-premier-guide-français-de-voyage-sort-un, consultées le 28.04.2011.

Baedeker, Karl, La Suisse et les parties limitrophes de la Savoie et de l'Italie. Manuel du voyageur, Leipzig, K. Baedeker éd., 24e éd., 1905.

Guide du Routard, Suisse, Paris, Hachette, 2006.

http://www.routard.com/planete_coulisse/page/saga.htm, consulté le 28.04.2011

Misson, François-Maximilien, Nouveau voyage d'Italie, 4 vol., Utrecht, Van De Water et Van Poolsum, 5e éd., 1722 (1691).

Reichard, Heinrich August Ottokar, Guide des voyageurs en Europe, 2 vols, Weimar, Bureau de l'Industrie, $1^{\text {ère }}$ éd., 1793, t. 1.

Stendhal, Journal, in Euvres intimes, éd. établie par Vittorio Del Litto, Paris, Gallimard, 2 vols, 1981 (La Pléiade).

\section{Travaux}

Certeau, Michel de, "Récits d'espace», in L'invention du quotidien, 1. Les arts de faire, Paris, Gallimard, 1990 (1980), p. 170-191.

Desportes, Marc, Paysages en mouvement. Transports et perception de l'espace, XVIII'-XX' siècle, Paris, Gallimard, 2005.

Devanthery, Ariane, Itinéraires. Les guides de voyage en Suisse de la fin $d u$ XVIII siècle à 1914. Contribution à une histoire culturelle du tourisme, thèse de doctorat, Université de Lausanne, 2008. 
—, «Entre espaces perçus et espaces à percevoir. Une lecture du trajet de Saint-Maurice à Martigny présenté par quatre guides de voyage (1788-1908)», Vallesia, 65 (2010), p. 155-188.

—, "Entre itinéraires et trajets, représentations des déplacements dans les guides de voyage au tournant du XIX' siècle", In Situ, revue des patrimoines, www.revue.inventaire.culture.gouv.fr, 2011. Consulté le 10.05.2011.

DewitTE, Jacques, «Espace du paysage et espace géographique. Examen critique de deux concepts d'Erwin Straus", in Lire l'espace, éd. par Jacques Poirier, Jean-Jacques Wunenburger, Bruxelles, Ousia, 1996, p. 313-328.

Meizoz, Jérôme, "Ethos et posture d'auteur (Rousseau, Céline, Ajar, Houellebecq)", in Sciences du texte et analyse de discours. Enjeux d'une interdisciplinarité, éd. par Jean-Michel Adam, Ute Heidmann, Etudes de Lettres, 270 (2005), p. 181-195.

Reichler, Claude, «Le marcheur romantique et la phénoménologie du chemin", in Marche et paysage. Les chemins de la géopoétique, dir. par Bertrand Lévy, Alexandre Gillet, Genève, Métropolis, 2007, p. 31-64. 
